\title{
Crossing Symmetric Dispersion Relations in Quantum Field Theories
}

\author{
Aninda Sinha $\odot^{*}$ and Ahmadullah Zahed $\odot^{\dagger}$ \\ Centre for High Energy Physics, Indian Institute of Science, C.V. Raman Avenue, Bangalore 560012, India
}

(Received 4 January 2021; accepted 15 April 2021; published 7 May 2021)

\begin{abstract}
For 2-2 scattering in quantum field theories, the usual fixed $t$ dispersion relation exhibits only twochannel symmetry. This Letter considers a crossing symmetric dispersion relation, reviving certain old ideas from the 1970s. Rather than the fixed $t$ dispersion relation, this needs a dispersion relation in a different variable $z$, which is related to the Mandelstam invariants $s, t, u$ via a parametric cubic relation making the crossing symmetry in the complex $z$ plane a geometric rotation. The resulting dispersion is manifestly three-channel crossing symmetric. We give simple derivations of certain known positivity conditions for effective field theories, including the null constraints, which lead to two sided bounds and derive a general set of new nonperturbative inequalities. We show how these inequalities enable us to locate the first massive string state from a low energy expansion of the four dilaton amplitude in type II string theory. We also show how a generalized (numerical) Froissart bound, valid for all energies, is obtained from this approach.
\end{abstract}

DOI: 10.1103/PhysRevLett.126.181601

Introduction.-Dispersion relations provide nonperturbative representations for scattering amplitudes in quantum field theories [1,2]. The usual way to write dispersion relations in the context of 2-2 scattering of identical particles is to keep one of the Mandelstam invariants, usually $t$, fixed and write a complex integral in the variable $s$. This approach naturally leads to an $s-u$ symmetric representation of the amplitude. Then, one imposes crossing symmetry as an additional condition. A similar approach can also be developed for Mellin amplitudes for conformal field theories. Recent developments in this direction include Refs. [3-5].

The amplitude's resulting representation not having manifest three-channel crossing symmetry may appear to be a drawback. For instance, in perturbative quantum field theories, when we compute Feynman diagrams, the amplitude's resulting expansion exhibits crossing symmetry. In the worldsheet formulation of string theory, the tree level sphere diagram, for instance, is also manifestly crossing symmetric. Hence, it seems like a natural question to ask as to how would one directly see the structure of Feynman diagrams from dispersion relations.

Is there a crossing symmetric version of the dispersion relations? In the 1970s, this question was briefly considered in a few papers, for example, in 1972 by Auberson and Khuri

Published by the American Physical Society under the terms of the Creative Commons Attribution 4.0 International license. Further distribution of this work must maintain attribution to the author(s) and the published article's title, journal citation, and DOI. Funded by SCOAP ${ }^{3}$. in Ref. [6] and in 1974 by Mahoux, Roy, and Wanders in Ref. [7]. Unfortunately, due to the technical complications involved, barring for a smattering of a few papers (e.g., Ref. [8]), this approach has not been well explored in the literature. We will follow Ref. [6] and revive this line of questioning again. In the CFT context, Polyakov's work in Ref. [9] proposed a fully crossing symmetric bootstrap, which was developed in Refs. [10,11]. However, this approach currently lacks a nonperturbative derivation for $d \geq 2$. Our methods in this Letter will enable us to address this important question in the near future [12].

Dispersion relations also give a window to understanding how analyticity and unitarity assumptions for the high energy behaviour of amplitudes constrain low energy physics contained in effective field theories (EFTs) [13]. Our manifestly crossing symmetric approach not only leads to a simpler and unifying derivation of recently considered positivity constraints in EFTs [14-17], but also enables us to write down a completely general set of positivity constraints on the Wilson coefficients. In particular, we will provide straightforward derivations of many of the upper bounds on the ratios of Wilson coefficients, as well as the null constraints listed in Refs. [16,17], leading to the lower bounds. Our formalism will enable us to write down general formulas for the upper bounds and the independent null constraints.

We will consider two novel applications of our constraints. First, using them, we will locate the first massive string pole from the low energy expansion for the tree-level four-dilaton scattering in type II string theory (e.g., Ref. [18]). Second, our approach enables us to derive a numerical upper bound on the total scattering cross section 
of identical particles valid at all energies, generalizing the famous Froissart bound [19]. Further, we will explain how our approach leads to a structure like the Feynman diagram expansion in QFTs.

Crossing symmetric dispersion relation.-We begin by considering cubic hypersurfaces [6] in the variables $s_{1}=s-\mu / 3, \quad s_{2}=t-\mu / 3, \quad s_{3}=u-\mu / 3=-s_{1}-s_{2}$, $\mu=4 m^{2}$, where $s, t, u$ are usual Mandelstam variables. Explicitly, these hypersurfaces will be given by $\left[s_{1}(z)-a\right]\left[s_{2}(z)-a\right]\left[s_{3}(z)-a\right]=-a^{3}$, with $a$ being a real parameter [20]. The $s_{i}$ 's can be parametrized via

$$
s_{k}=a-\frac{a\left(z-z_{k}\right)^{3}}{z^{3}-1}, \quad k=1,2,3,
$$

where $z_{k}$ are cube roots of unity and we will restrict $-\mu / 3 \leq a<2 \mu / 3$. In Ref. [6], it is shown that the amplitude is analytic in the interval $-6.71 \mu<a<2 \mu / 3$. Importantly, note that $a=\left(s_{1} s_{2} s_{3}\right) /\left(s_{1} s_{2}+s_{2} s_{3}+s_{3} s_{1}\right)$ is crossing symmetric in the usual variables. The amplitude $\mathcal{M}\left(s_{1}, s_{2}\right)$ can be written as an analytic function of $(z, a)$, i.e., $\quad \overline{\mathcal{M}}(z, a)=\mathcal{M}\left[s_{1}(z), s_{2}(z)\right] . \quad \mathcal{M}\left[s_{1}(z), s_{2}(z)\right]$ has physical cuts for $s_{k} \geq 2 \mu / 3, k=1,2,3$. These physical cuts get mapped to portions of arcs on the unit circle in the complex $z$ plane. We can write down a twice subtracted dispersion relation $z$ variable, for fixed $a$. For the completely crossing symmetric case, the dispersion relation simplifies dramatically in terms of the $s_{1}, s_{2}, s_{3}$ variables:

$$
\begin{aligned}
\mathcal{M}_{0}\left(s_{1}, s_{2}\right)= & \alpha_{0}+\frac{1}{\pi} \int_{2 \mu / 3}^{\infty} \frac{d s_{1}^{\prime}}{s_{1}^{\prime}} \mathcal{A}\left[s_{1}^{\prime} ; s_{2}^{(+)}\left(s_{1}^{\prime}, a\right)\right] \\
& \times H\left(s_{1}^{\prime} ; s_{1}, s_{2}, s_{3}\right),
\end{aligned}
$$

where $\mathcal{A}\left(s_{1} ; s_{2}\right)$ is the $s$-channel discontinuity and

$$
\begin{aligned}
H\left(s_{1}^{\prime} ; s_{1}, s_{2}, s_{3}\right) & =\left[\frac{s_{1}}{\left(s_{1}^{\prime}-s_{1}\right)}+\frac{s_{2}}{\left(s_{1}^{\prime}-s_{2}\right)}+\frac{s_{3}}{\left(s_{1}^{\prime}-s_{3}\right)}\right], \\
s_{2}^{(+)}\left(s_{1}^{\prime}, a\right) & =-\frac{s_{1}^{\prime}}{2}\left[1-\left(\frac{s_{1}^{\prime}+3 a}{s_{1}^{\prime}-a}\right)^{1 / 2}\right],
\end{aligned}
$$

which defines the crossing symmetric kernel $H$ [21]. $\alpha_{0}$ is a subtraction constant. From hereon, we will follow a more direct route than what was followed in Ref. [6]. This final form in Eq. (2) is manifestly three channel crossing symmetric. Notice the nontrivial $s_{2}^{(+)}$dependence in $\mathcal{A}$-we emphasize that the crossing symmetric $s_{1}, s_{2}, s_{3}$ dependence also comes via $s_{2}^{(+)}$through $a$. One can use the crossing symmetric form and carry out several checks. For instance, we have numerically investigated how well the representation represents the type II superstring four dilaton amplitude [21]. For this case, on the real $\left(s_{1}, s_{2}\right)$ plane, the crossing symmetric formula is often a better representation than the conventional dispersion relation. For the situation where we have no massless poles, or where we subtract them out, the crossing symmetric amplitude has an expansion

$$
\mathcal{M}_{0}\left(s_{1}, s_{2}\right)=\sum_{p, q=0}^{\infty} \mathcal{W}_{p q} x^{p} y^{q}
$$

with $x=-\left(s_{1} s_{2}+s_{2} s_{3}+s_{3} s_{1}\right), y=-s_{1} s_{2} s_{3}$. Note that $H\left(s_{1}^{\prime} ; s_{1}, s_{2}, s_{3}\right)=\left[x\left(2 s_{1}^{\prime}-3 a\right)\right] /\left[x a-x s_{1}^{\prime}+s_{1}^{\prime 3}\right]$, which can be seen by writing $s_{k}$ 's in terms of $(z, a)$ and identifying $z^{3} /\left(z^{3}-1\right)^{2}=-x / 27 a^{2}$. We can now expand Eq. (2) in powers of $x, a$ using the $s$-channel discontinuity which has a partial wave expansion in terms of Gegenbauer polynomials involving even spins

$$
\begin{aligned}
\mathcal{A}\left(s_{1}, s_{2}^{(+)}\left(s_{1}, a\right)\right) & =\Phi\left(s_{1}\right) \sum_{\ell=0}^{\infty}(2 \ell+2 \alpha) a_{\ell}\left(s_{1}\right) C_{\ell}^{(\alpha)}\left(\sqrt{\xi\left(s_{1}, a\right)}\right), \\
\xi\left(s_{1}, a\right) & =\xi_{0}+4 \xi_{0}\left(\frac{a}{s_{1}-a}\right), \quad \xi_{0}=\frac{s_{1}^{2}}{\left(s_{1}-2 \mu / 3\right)^{2}},
\end{aligned}
$$

where $\quad \alpha=(d-3) / 2, \quad \Phi\left(s_{1}\right)=\Psi(\alpha)\left[\sqrt{s_{1}+\mu / 3} /\right.$ $\left.\left(s_{1}-2 \mu / 3\right)^{\alpha}\right]$, and $\Psi(\alpha)$ is real positive number. Expanding the Gegenbauer polynomials around $\xi=\xi_{0}$, with $p_{\ell}^{(j)}\left(\xi_{0}\right)=\partial^{j} C_{\ell}^{(\alpha)}(\sqrt{\xi}) /\left.\partial \xi^{j}\right|_{\xi=\xi_{0}}$, we find the coefficient of $a^{m}$ leading to an inversion formula

$$
\begin{aligned}
\mathcal{W}_{n-m, m} & =\int_{\frac{2 \mu}{3}}^{\infty} \frac{d s_{1}}{s_{1}} \Phi\left(s_{1}\right) \sum_{\ell=0}^{\infty}(2 \ell+2 \alpha) a_{\ell}\left(s_{1}\right) \mathcal{B}_{n, m}^{(\ell)}\left(s_{1}\right), \\
\mathcal{B}_{n, m}^{(\ell)}\left(s_{1}\right) & =\sum_{j=0}^{m} \frac{p_{\ell}^{(j)}\left(\xi_{0}\right)\left(4 \xi_{0}\right)^{j}(3 j-m-2 n)(-n)_{m}}{\pi s_{1}^{2 n+m} j !(m-j) !(-n)_{j+1}}
\end{aligned}
$$

for $n \geq 1$. Note that $\mathcal{W}_{0,0}=\alpha_{0}$. Equation (4) allows two lines of investigation. (a) For $n \geq m$ with $n \geq 1$, we get the coefficients in terms of $a_{\ell}\left(s_{1}\right)$. Since partial wave unitarity implies $0 \leq a_{\ell}\left(s_{1}\right) \leq 1$, we can find positivity constraints on $\mathcal{W}_{n-m, m}$. (b) For $n<m$ with $n \geq 1$, the coefficients should vanish, as needed by Eq. (3), which give rise to nontrivial constraints on $a_{\ell}\left(s_{1}\right)$. Notice that since $\ell$ is even, we will need $\ell \geq 2 n$. These sum rules or "null constraints" are instrumental in getting the lower bounds on $\mathcal{W}_{n-m, m}$ in EFTs. We will use these sum rules to put a bound on the total cross section at any $s_{1}$, generalizing the famous Froissart bound. 
Constraining QFTs.-Now from Eq. (4), we can derive inequalities involving $\mathcal{W}_{p, q}$. We use the unitarity constraints $0 \leq a_{\ell}\left(s_{1}\right) \leq 1$ as well as $C_{\ell-k}^{(\alpha+k)}(2 \mu / 3 \delta+1) \geq 0$, for $\delta=s_{1}-2 \mu / 3 \geq 0, \alpha \geq 0, \mu \geq 0$. Since the range of $s_{1}$ in Eq. (4) starts at $2 \mu / 3$, we have introduced $\delta$ as a convenient variable. We have $\sqrt{\xi_{0}}=2 \mu / 3 \delta+1$. Note that $p_{\ell}^{(j)}$, s involve derivatives of Gegenbauer. Specifically we find the useful expression

$$
\mathcal{B}_{n, m}^{(\ell)}\left(s_{1}\right)=\sum_{k=0}^{m} \mathfrak{U}_{n, m, k}^{(\alpha)}(-1)^{k+m} C_{\ell-k}^{(\alpha+k)}\left(\frac{2 \mu}{3 \delta}+1\right),
$$

where [22]

$$
\mathfrak{U}_{n, m, k}^{(\alpha)}=\sum_{j=k}^{m} \frac{{\sqrt{16 \xi_{0}}}^{k}(\alpha)_{k}(m+2 n-3 j) \Gamma(n-j) \Gamma(2 j-k)}{s_{1}^{m+2 n} \Gamma(k) j !(m-j) !(j-k) !(n-m) !},
$$

is positive for $n \geq m$. Note that in the sum, $(-1)^{k+m}$ spoils the definite sign of $\mathcal{B}_{n, m}^{(\ell)}\left(s_{1}\right)$. We can search for $\chi_{n}^{(r, m)}(\mu, \delta)$, such that

$$
\sum_{r=0}^{m} \chi_{n}^{(r, m)}(\mu, \delta) \mathcal{B}_{n, r}^{(\ell)}\left(s_{1}\right)=\mathfrak{U}_{n, m, m}^{(\alpha)} C_{\ell-m}^{(\alpha+m)}\left(\frac{2 \mu}{3 \delta}+1\right) \geq 0
$$

for $m \leq n$. A solution is easily found using the following recursion relation:

$$
\chi_{n}^{(r, m)}(\mu, \delta)=\sum_{j=r+1}^{m}(-1)^{j+r+1} \chi_{n}^{(j, m)} \frac{\mathfrak{U}_{n, j, r}^{(\alpha)}\left(\frac{2 \mu}{3}+\delta\right)}{\mathfrak{U}_{n, r, r}^{(\alpha)}\left(\frac{2 \mu}{3}+\delta\right)},
$$

with $\chi_{n}^{(m, m)}(\mu, \delta)=1$. Using the recursion relation, one can check that [23] $\chi_{n}^{(r, m)}>0$, for $n \geq m$. Then Eq. (4) leads to [24]

$$
\sum_{r=0}^{m} \chi_{n}^{(r, m)}(\mu, \delta=0) \mathcal{W}_{n-r, r} \geq 0
$$

which we will refer to as nonperturbative constraints to differentiate from the EFT constraints to be derived next. In order to derive EFT bounds, we start with Eq. (4) and write

$\mathcal{W}_{n-m, m}^{\left(\delta_{0}\right)} \equiv \int_{\delta_{0}+2 \mu / 3}^{\infty} \frac{d s_{1}}{s_{1}} \Phi\left(s_{1}\right) \sum_{\ell=0}^{\infty}(2 \ell+2 \alpha) a_{\ell}\left(s_{1}\right) \mathcal{B}_{n, m}^{(\ell)}\left(s_{1}\right)$

for $n \geq 1$, which defines for us the Wilson coefficients and are the $\mathcal{W}$ 's in Eq. (4) when $\delta_{0}=0$. In such cases, we can show that

$$
\sum_{r=0}^{m} \chi_{n}^{(r, m)}\left(\mu, \delta_{0}\right) \mathcal{B}_{n, r}^{(\ell)}\left(\delta+\frac{2 \mu}{3}\right) \geq 0
$$

for $\delta \geq \delta_{0}$. This leads to positivity constraints

$$
\sum_{r=0}^{m} \chi_{n}^{(r, m)}\left(\mu, \delta=\delta_{0}\right) \mathcal{W}_{n-r, r}^{\left(\delta_{0}\right)} \geq 0,
$$

for $\quad \mu \geq 0, \quad \delta_{0} \geq 0, \quad m \leq n$. Since $\mathcal{B}_{n, 0}^{(\ell)}(\delta+2 \mu / 3)=$ $\left\{\left[2 C_{\ell}^{(\alpha)}(2 \mu / 3 \delta+1)\right] /\left[\pi(\delta+2 \mu / 3)^{2 n}\right]\right\}$, we have

$$
\mathcal{W}_{n, 0}^{\left(\delta_{0}\right)} \leq \frac{1}{\left(\delta_{0}+\frac{2 \mu}{3}\right)^{2}} \mathcal{W}_{n-1,0}^{\left(\delta_{0}\right)} .
$$

Our expressions are in agreement with the limited number of cases known in the literature $[14,16,17]$ except that our derivation is manifestly crossing symmetric from the start and admit a straightforward generalization [25].

An immediate application of our general formulas is the examination of the $n \gg m$ limit. We find simply

$$
\sum_{r=0}^{m} \frac{n^{m-r}}{(m-r) !\left(\delta_{0}+\frac{2 \mu}{3}\right)^{m-r}} \frac{\mathcal{W}_{n-r, r}^{\left(\delta_{0}\right)}}{\mathcal{W}_{n, 0}^{\left(\delta_{0}\right)}} \geq 0,
$$

for $n \gg m$. We have checked that tree level type II string theory, to be discussed below, respects this.

Null constraints. - To derive lower bounds, we make use of the $n<m$ vanishing conditions arising from Eq. (4). In the large $\delta$ limit, we have [26]

$$
\begin{aligned}
\mathcal{B}_{n, m}^{(\ell)}(\delta) & =\frac{C_{\ell}^{(\alpha)}(1)}{\pi} \frac{D_{\ell, \alpha}^{(n, m)}}{\delta^{2 n+m}}+O\left(\frac{\mu}{\delta^{2 n+m+1}}\right), \\
D_{\ell, \alpha}^{(n, m)} & =\sum_{j=0}^{m} \frac{(-4)^{j}\left(-\frac{\ell}{2}\right)_{j}\left(\alpha+\frac{\ell}{2}\right)_{j}}{\left(\alpha+\frac{1}{2}\right)_{j}} \frac{(3 j-m-2 n) \Gamma(m-n)}{j !(m-j) !(j-n) !} .
\end{aligned}
$$

Then in the limit when $\delta_{0} \gg \mu$ we have [27]

$\int_{\delta_{0}}^{\infty} \frac{d s_{1}}{s_{1}^{\alpha+1 / 2}} \sum_{\ell=2}^{\infty}(2 \ell+2 \alpha) a_{\ell}\left(s_{1}\right) \frac{C_{\ell}^{(\alpha)}(1)}{\pi} \frac{D_{\ell, \alpha}^{(n, m)}}{s_{1}^{2 n+m}}=0$.

for $m>n, n \geq 1$. For example $m=2, n=1$ gives $D_{\ell, \alpha}^{(1,2)}=$ $\{2 \ell(\ell+2 \alpha)[-11-10 \alpha+2 \ell(\ell+2 \alpha)] /(2 \alpha+1)(2 \alpha+3)\}$, which was first derived in Ref. [16] from fixed- $t$ dispersion relations [28]. Once these null constraints are in place, a judicious use of Cauchy-Schwarz inequality as used in Ref. [16], or a more constraining numerical argument used in Ref. [17] can be pursued to derive lower bounds. The existence of such bounds was originally emphasized in Ref. [29]. Our approach gives completely general expressions for the independent null constraints.

Applications.-We will now consider two applications. The first application will make use of the $n \geq m$ constraints while the second will use the $n<m$ constraints arising from Eq. (4). 
Tree level type II superstring theory.-The four dilaton type II superstring tree amplitude is given by [18]

$$
\mathcal{M}\left(s_{1}, s_{2}\right)=-\frac{\Gamma\left(-s_{1}\right) \Gamma\left(-s_{2}\right) \Gamma\left(s_{1}+s_{2}\right)}{\Gamma\left(s_{1}+1\right) \Gamma\left(-s_{1}-s_{2}+1\right) \Gamma\left(s_{2}+1\right)} .
$$

We consider the amplitude $\mathcal{M}^{(c l)}$ obtained after subtracting out the massless pole $-1 /\left[s_{1} s_{2}\left(s_{1}+s_{2}\right)\right]$. We can easily compute the $\mathcal{W}_{p, q}$, for example [18], $\mathcal{W}_{0,0}=2 \zeta(3)$, $\mathcal{W}_{0,1}=-2 \zeta(3)^{2}, \mathcal{W}_{0,2}=\frac{2}{3}\left[2 \zeta(3)^{3}+\zeta(9)\right], \mathcal{W}_{1,0}=2 \zeta(5)$, $\mathcal{W}_{1,1}=-4 \zeta(3) \zeta(5)$. Suppose we are given the first few terms in the derivative expansion. Then, can we say where the first massive string pole would occur? More precisely in Eq. (6), what is the maximum $\delta_{0}$ we can use? Using the methods in this Letter, we can address this question.

From $m=1, n=1$ (6 derivatives) condition (8) $(\mu=0)$ gives us $\left[3 \zeta(5) / \delta_{0}\right]-2 \zeta(3)^{2}>0$, which implies $\delta_{0}<1.07644$. Similarly from other conditions, we can show that $\delta_{0}<\delta_{0}^{(\max )}$. In Fig. 1, we have shown that for higher constraints [30], the $\delta_{0}^{(\max )}$ converges towards 1 , which is exactly the location of the first massive pole.

Bound on total scattering cross section.-Now, we will exploit the null constraints arising from $m>n$ in Eq. (4) to bound total scattering cross sections. We will be in $d=4$ or $\alpha=1 / 2$ and we will use the standard notation $s=s_{1}+$ $4 / 3$ with $\mu=4$. The null constraints read

$\int_{4}^{\infty} \frac{d s}{s-\frac{4}{3}} \Phi(s) \sum_{\ell=2 n}^{\infty}(2 \ell+1) a_{\ell}(s) \mathcal{B}_{m, n}^{(\ell)}\left(s-\frac{4}{3}\right)=0$,

where $\Phi(s)=\sqrt{s /(s-4)}$. for $m>n, \quad n \geq 1$. For $m+n \leq \ell, \quad m>n, \quad n \geq 1 \quad$ we can verify that $\mathcal{B}_{m, n}^{(\ell)}\left(s-\frac{4}{3}\right) \geq 0$. Then we can write (12) as

$$
\int_{4}^{\infty} \frac{d s}{s-\frac{4}{3}} \Phi(s) \sum_{\ell=2 n}^{L_{\max }}(2 \ell+1) a_{\ell}(s) \mathcal{B}_{m, n}^{(\ell)}\left(s-\frac{4}{3}\right) \leq 0,
$$

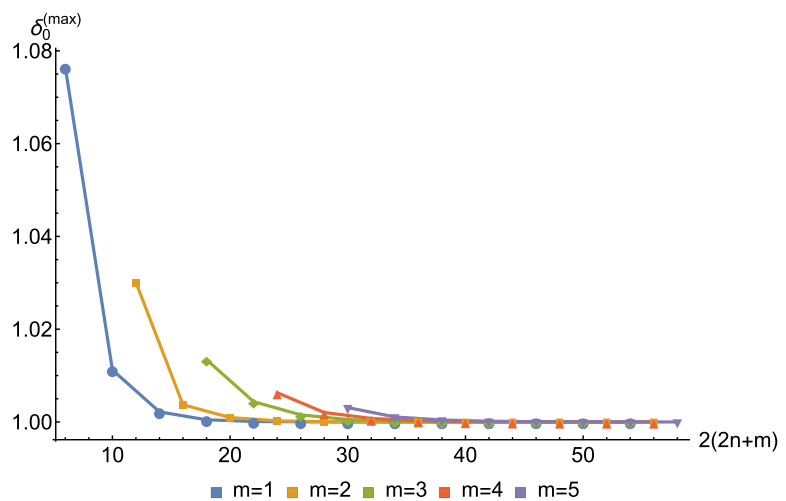

FIG. 1. $\delta_{0}^{(\max )}$ vs $2(2 n+m)$, the derivative order, for $m=1,2$, $3,4,5$. for $m+n \leq L_{\max }, m>n, n \geq 1$. We have placed the contributions arising from $\ell \geq L_{\max }+2$ on the right which gives the inequality. From unitarity, we know that $0 \leq a_{\ell}(s) \leq 1$. The inequalities (13) impose further conditions on the $a_{\ell}(s)$. We convert the integral over $s$ in Eq. (13) as a sum by defining $s(k)=4+k\left[\left(s_{\max }-4\right) / N_{\max }\right]$. Using the constraints (13), we want to bound the total scattering cross section $\sigma(s)[31]$

$\sigma(s)=\frac{16 \pi}{s-4} \sum_{\ell=0}^{\infty}(2 \ell+1) a_{\ell}(s) \approx \frac{16 \pi}{s-4} \sum_{\ell=0}^{L_{\max }}(2 \ell+1) a_{\ell}(s)$.

We maximize the value of $(s-4) / 16 \pi \times \sigma(s)=$ $\bar{\sigma}=\sum_{\ell=0}^{L_{\max }}(2 \ell+1) a_{\ell}(s)$. The bound is shown in Fig. 2 for various [34] $L_{\max }=36,46,60$. A fit with $\bar{\sigma}_{0} \times$ $s \log ^{2}\left(s / s_{0}\right)$ can be found. The $L_{\max }=36$ gives fit values $\bar{\sigma}_{0}=0.29, s_{0}=0.128$, similarly $L_{\max }=60$ gives fit values $\bar{\sigma}_{0}=0.22, s_{0}=0.066$. The convergence with the spin sum is suggested by the figure but appears to be slow for higher values of $s$. Also it is clear that the $\bar{\sigma}$ found using a typical $S$ matrix living on the boundary of the so-called river arising from the $S$-matrix bootstrap [35] or even the lake boundary in Ref. [36], is far below the numerical bound presented.

Note that Froissart bound [37], i.e., $\bar{\sigma} \lesssim(s / 16) \log ^{2}\left(s / s_{0}\right)$ (which is not valid for lower value of $s$ we are considering) is below the numerical bound. The main utility of our numerical bound is that it is valid for any $s \geq 4$ unlike the Froissart bound which is valid for $s \gg 4$. It will be fascinating to derive analytic bounds using the present method and see if a stronger than Froissart bound is possible at higher energies. In existing derivations of the Froissart bound, the role of crossing symmetry has not been explored.

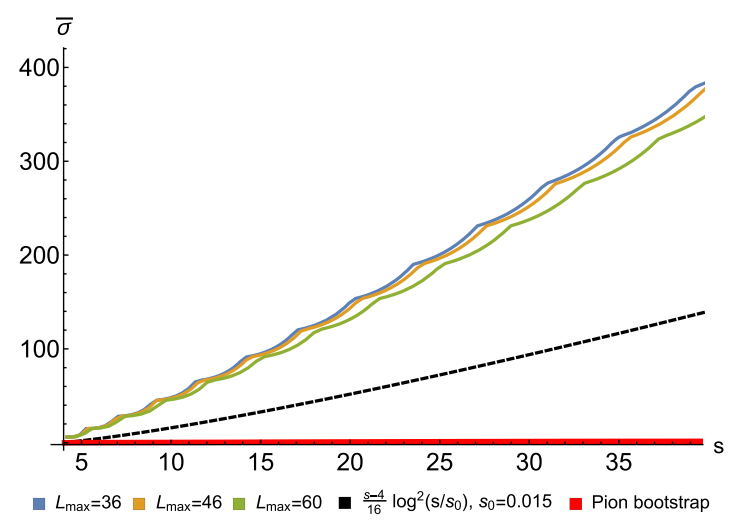

FIG. 2. The bound on $\bar{\sigma}(s)$ using constraints (13) with $L_{\max }=36,46,60$. The black dashed line is the Froissart bound on $\bar{\sigma}$. The red line is maximum $\bar{\sigma}$ obtained using the pion bootstrap in Ref. [35]. 
We derive a simple analytic bound in the Supplemental Material [21].

Feynman block expansion of amplitude.-We will now address how the structure of the Feynman diagram expansion emerges from our analysis. Given the partial wave expansion of the $s$-channel discontinuity, we can write the amplitude as

$$
\begin{aligned}
\mathcal{M}\left(s_{1}, s_{2}\right)= & \alpha_{0}+\frac{1}{\pi} \int_{2 \mu / 3}^{\infty} \sum_{\ell=0}^{\infty} \frac{d \sigma}{\sigma} H\left(\sigma ; s_{1}, s_{2}, s_{3}\right) \\
& \left(\Phi(\sigma ; \alpha)(2 \ell+2 \alpha) \frac{a_{\ell}(\sigma)}{\left(\sigma-\frac{2 \mu}{3}\right)^{\ell}} Q_{\ell}\left(\sigma ; s_{2}^{(+)}(\sigma, a)\right)\right),
\end{aligned}
$$

where $Q_{\ell}\left(s_{1}, s_{2}\right)=\left(s_{1}-2 \mu / 3\right)^{\ell} C_{\ell}^{(\alpha)}\left[1+2\left(s_{2}+\mu / 3\right) /\right.$ $\left(s_{1}-2 \mu / 3\right)$ ]. Let us refer to $H Q_{\ell}$ as the Dyson blocknote that this block has nonlocal negative powers of $x$ which should cancel on using the null constraints. A natural way to proceed would be to use a basis which has these spurious negative powers of $x$ removed at the onset. This leads us to define the Feynman block

$$
M_{\ell}^{F}\left(\sigma ; s_{1}, s_{2}\right)=\sum_{i=1}^{3} M_{\ell}^{(i)}\left(\sigma ; s_{1}, s_{2}\right)+M_{\ell}^{(c)}\left(\sigma ; s_{1}, s_{2}\right),
$$

where the $s$-channel part of the Feynman block is

$$
M_{\ell}^{(1)}\left(\sigma ; s_{1}, s_{2}\right)=Q_{\ell}\left(s_{1}, s_{2}\right)\left(\frac{1}{\sigma-s_{1}}-\frac{1}{\sigma}\right) .
$$

The $t, u$ channel are given by $M_{\ell}^{(2)}\left(\sigma ; s_{1}, s_{2}\right)=M_{\ell}^{(1)}\left(\sigma ; s_{2}, s_{3}\right)$, $M_{\ell}^{(3)}\left(\sigma ; s_{1}, s_{2}\right)=M_{\ell}^{(1)}\left(\sigma ; s_{3}, s_{1}\right) . M_{\ell}^{(c)}\left(\sigma ; s_{1}, s_{2}\right)$ are contact terms involving polynomials of $s_{i}$ 's. To find this write

$\mathcal{D}_{\ell}(\sigma)=\frac{1}{\sigma} Q_{\ell}\left(\sigma, s_{2}^{(+)}\right) H\left(\sigma ; s_{1}, s_{2}, s_{3}\right)-\sum_{i=1}^{3} M_{\ell}^{(i)}\left(\sigma ; s_{1}, s_{2}\right)$.

For example, $\quad \mathcal{D}_{\ell=2}(\sigma)=\left[\left(x b_{0,2}^{(2)}\right) /(a-\sigma)\right]+\left[x\left(b_{0,2}^{(2)}+\right.\right.$ $\left.\left.2 b_{2,0}^{(2)}\right)\right] / \sigma=2 x b_{2,0}^{(2)} / \sigma-y b_{0,2}^{(2)} /(\sigma)^{2}-\left(x b_{0,2}^{(2)}\right) \sum_{n=2}^{\infty} a^{n}(\sigma)^{-n-1}$, where $b_{n, m}^{(\ell)}=(1 / n ! m !) \partial_{s_{1}}^{n} \partial_{s_{2}}^{m}\left[Q_{\ell}\left(s_{1}, s_{2}\right)\right]$. We throw away negative powers in $x$ (nonlocal terms), which gives

$$
M_{\ell=2}^{(c)}\left(\sigma ; s_{1}, s_{2}\right)=\frac{2 x b_{2,0}^{(2)}}{\sigma}-\frac{y b_{0,2}^{(2)}}{\sigma^{2}},
$$

which is the polynomial form of the contact term. This can be repeated for any $\ell$. In general, we can write the Feynman block expansion of an amplitude

$$
\begin{aligned}
\mathcal{M}\left(s_{1}, s_{2}\right)= & \alpha_{0}+\frac{1}{\pi} \sum_{\ell=0}^{\infty} \int_{2 \mu / 3}^{\infty} \frac{d \sigma}{\left(\sigma-\frac{2 \mu}{3}\right)^{\ell}} \\
& \times\left(\Phi(\sigma ; \alpha)(2 \ell+2 \alpha) a_{\ell}(\sigma) M_{\ell}^{F}\left(\sigma ; s_{1}, s_{2}\right)\right) .
\end{aligned}
$$

This demonstrates how the structure of the Feynman diagram expansion, involving exchanges and contact diagrams, emerges from the dispersion relation. Equation (15) converges for $-6.71 \mu<a<2 \mu / 3$ [6]. Thus, expanding Eq. (15) around $a=0$ gives a convergent representation in $a^{n} x^{m}$ powers. The difference between Eqs. (17) and (15) are the terms $m<n$ in the latter. Removing these will leave us with a convergent representation (17) around $a=0$ see Supplemental Material [21] for further checks.

Discussion.-The crossing symmetric dispersion relation approach, presented in this Letter, promises to open up a new and efficient way to study field theories. We saw how the picture of Feynman diagrams emerges from the crossing symmetric approach; seeing this using the fixed- $t$ dispersion relation is impossible as crossing symmetry is imposed as a constraint. It will be very interesting to connect the ideas and techniques in this Letter with the "EFT-hedron" picture in Ref. [39]. Another place where we expect these crossing symmetric dispersion relations to play an important role [40] is the formulation of the dual $S$-matrix bootstrap in higher dimensions. So far, an explicit attempt has only been made in two dimensions [41].

On the CFT side, we have shown in Ref. [12] how the manifestly crossing symmetric method extended to CFT Mellin amplitudes leads to the sum rule constraints arising from the two-channel dispersion relation presented in Refs. [3,4]. Furthermore, the CFT generalized null constraints admit a straightforward derivation and are needed to show the equivalence. This suggests that the manifestly crossing symmetric dispersion relation will not only be more systematic but will have more constraints than what is easily derivable in the two-channel symmetric approach.

We thank Parijat Dey, Kausik Ghosh, Parthiv Haldar, and Apratim Kaviraj for useful discussions. Special thanks to Andrew Tolley for comments on the draft and to Yu-tin Huang for comments on the draft and sharing [39]. We especially thank Rajesh Gopakumar for numerous discussions and encouragement for this work, comments on the draft, as well as for ongoing collaboration in Ref. [12].

*asinha@iisc.ac.in †ahmadullah@iisc.ac.in

[1] S. Mandelstam, Determination of the pion-nucleon scattering amplitude from dispersion relations and unitarity. General theory, Phys. Rev. 112, 1344 (1958).

[2] H.M Nussenzveig, Causality and Dispersion Relations (New York publishers, 1972).

[3] J. Penedones, J. A. Silva, and A. Zhiboedov, Nonperturbative mellin amplitudes: Existence, properties, applications, J. High Energy Phys. 08 (2020) 031.

[4] D. Carmi, J. Penedones, J. A. Silva, and A. Zhiboedov, Applications of dispersive sum rules: $\epsilon$-expansion and holography, arXiv:2009.13506.

[5] S. Caron-Huot, D. Mazac, L. Rastelli, and D. SimmonsDuffin, Dispersive CFT sum rules, arXiv:2008.04931. 
[6] G. Auberson and N. N. Khuri, Rigorous parametric dispersion representation with three-channel symmetry, Phys. Rev. D 6, 2953 (1972).

[7] G. Mahoux, S. M. Roy, and G. Wanders, Physical pion pion partial-wave equations based on three channel crossing symmetry, Nucl. Phys. B70, 297 (1974).

[8] B. Ananthanarayan, The Low-energy expansion for pion pion scattering and crossing symmetry in dispersion relations, Phys. Rev. D 58, 036002 (1998).

[9] A. M. Polyakov, Nonhamiltonian approach to conformal quantum field theory, Zh. Eksp. Teor. Fiz. 66, 23 (1974) [Sov. Phys. JETP 39, 0 (1974)], https://inspirehep.net/ literature/96660.

[10] K. Sen and A. Sinha, On critical exponents without Feynman diagrams, J. Phys. A 49, 445401 (2016).

[11] R. Gopakumar, A. Kaviraj, K. Sen, and A. Sinha, Conformal Bootstrap in Mellin Space, Phys. Rev. Lett. 118, 081601 (2017); A Mellin space approach to the conformal bootstrap, J. High Energy Phys. 05 (2017) 027; R. Gopakumar and A. Sinha, On the Polyakov-Mellin bootstrap, J. High Energy Phys. 12 (2018) 040.

[12] R. Gopakumar, A. Sinha, and A. Zahed, Crossing symmetric dispersion relations for Mellin amplitudes, arXiv: 2101.09017.

[13] A. Adams, N. Arkani-Hamed, S. Dubovsky, A. Nicolis, and R. Rattazzi, Causality, analyticity and an IR obstruction to UV completion, J. High Energy Phys. 10 (2006) 014.

[14] C. de Rham, S. Melville, A. J. Tolley, and S. Y. Zhou, Positivity bounds for scalar field theories, Phys. Rev. D 96, 081702(R) (2017).

[15] B. Bellazzini, J. Elias Miró, R. Rattazzi, M. Riembau, and F. Riva, Positive moments for scattering amplitudes, arXiv:2011.00037.

[16] A. J. Tolley, Z. Y. Wang, and S. Y. Zhou, New positivity bounds from full crossing symmetry, arXiv:2011.02400.

[17] S. Caron-Huot and V. Van Duong, Extremal effective field theories, arXiv:2011.02957.

[18] M. B. Green and C. Wen, Superstring amplitudes, unitarily, and Hankel determinants of multiple zeta values, J. High Energy Phys. 11 (2019) 079.

[19] M. Froissart, Asymptotic behavior and subtractions in the Mandelstam representation, Phys. Rev. 123, 1053 (1961).

[20] The enlarged domain of $a$ is worked out in Ref. [6].

[21] See Supplemental Material at http://link.aps.org/ supplemental/10.1103/PhysRevLett.126.181601 for useful algebraic details.

[22] A closed form can be found in terms of a ${ }_{4} F_{3}$ hypergeometric function.

[23] Explicitly, for the first few cases, we have $\chi_{n}^{(0,1)}(\mu, \delta)=\frac{6 n+3}{6 \delta+4 \mu}, \quad \chi_{n}^{(0,2)}(\mu, \delta)=\{[9(2 n(n+2)+3)] /$ $\left.\left[4(3 \delta+2 \mu)^{2}\right]\right\}, \chi_{n}^{(1,2)}(\mu, \delta)=(6 n+3) /(6 \delta+4 \mu)$.

[24] The solutions for $\chi$ are unique in the sense that instead of $C_{\ell-k}^{(\alpha)}$, one can use positivity properties $p_{\ell}^{(j)}\left(\xi_{0}\right)$ itself which is proportional to the Jacobi polynomial $P_{\ell / 2-j}^{(-\ell-\alpha,-1 / 2+j+\alpha)}\left(1-2 / \xi_{0}\right)$. In this case, the $\chi$ 's are linear combinations of our solutions presented. In the Supplemental Material [21] we find the extremal solution to these inequalities.

[25] The $n=2$ case of Eq. (17) for $\delta_{0} \gg \mu$, which gives [17] [Eq. (4.2)]. The $m=1, n=1$ case of the Eq. (16) for $\delta_{0} \gg \mu$ was first derived in Ref. [14] [Eq. (29)] and the $m=1, n=1,2$ cases can be found in Ref. [16] [Eq. (6.1)] and [17] [Eq. (4.2)]. The $m=2, n=2$ case of Eq. (16) is given in Ref. [16] [1st equation of (6.2)], with the use of $n=3$ in Eq. (17).

[26] A closed form exists in terms of a ${ }_{4} F_{3}$ hypergeometric function.

[27] In the large $\ell$ limit, $C_{\ell}^{(\alpha)}(1) D_{\ell, \alpha}^{(n, m)} \sim \ell^{2 m+2 \alpha-1}$. Convergence of the sum in Eq. (21) implies $a_{\ell} \sim 1 / \ell^{2 m+2 \alpha+2}, \forall m$. Therefore, $a_{\ell}$ is "superpolynomially suppressed" in $\ell$.

[28] Some of the null constraints listed in Ref. [17] [Eq. (3.29)] are related to $D_{\ell, \alpha}^{(n, m)}$ via $D_{\ell, \alpha}^{(1,2)}=n_{4} / 2\left(\alpha+\frac{1}{2}\right)_{2}, D_{\ell, \alpha}^{(1,3)}=$ $n_{5} / 12\left(\alpha+\frac{1}{2}\right)_{3}, \quad D_{\ell, \alpha}^{(1,4)}=n_{6} / 24\left(\alpha+\frac{1}{2}\right)_{4}, \quad D_{\ell, \alpha}^{(1,5)}-D_{\ell, \alpha}^{(2,3)}=$ $n_{7} / 240\left(\alpha+\frac{1}{2}\right)_{5}$.

[29] N. Arkani-Hamed and Y.-T. Huang, Lectures at the CERN Winter School on Supergravity, Strings and Gauge Theory (2019).

[30] For example $m=3, \quad n=4 \quad$ (22 derivatives), $\delta_{0}^{(\max )}=1.00437$, for $m=1, n=8 \quad(34$ derivatives $)$, $\delta_{0}^{(\max )}=1.000000833$.

[31] See Refs. [32,33] for conventions. We are truncating the spin sum to $L_{\max }$, assuming higher spins are suppressed.

[32] A. Martin, Scattering theory: Unitarity, analyticity and crossing, Lect. Notes Phys. 3, 1 (1969).

[33] P. Haldar and A. Sinha, Froissart bound for/from CFT Mellin amplitudes, SciPost Phys. 8, 095 (2020).

[34] For $L_{\max }=36, s_{\max }=90, N_{\max }=480$, for $L_{\max }=46$, $s_{\max }=100, \quad N_{\max }=500, \quad$ for $L_{\max }=60, \quad s_{\max }=130$, $N_{\max }=550$.

[35] A. Bose, P. Haldar, A. Sinha, P. Sinha, and S. S. Tiwari, Relative entropy in scattering and the S-matrix bootstrap, SciPost Phys. 9, 081 (2020); A. Bose, A. Sinha, and S. S. Tiwari, Selection rules for the S-Matrix bootstrap, arXiv:2011.07944.

[36] A. L. Guerrieri, J. Penedones, and P. Vieira, Bootstrapping QCD Using Pion Scattering Amplitudes, Phys. Rev. Lett. 122, 241604 (2019).

[37] We have used $s_{0}=0.015$ in the Froissart bound plot in Fig. 2. This is the minimum possible value of $s_{0}$, see Ref. [38].

[38] A. Martin and S. M. Roy, Froissart bound on total crosssection without unknown constants, Phys. Rev. D 89, 045015 (2014).

[39] N. Arkani-Hamed, T. Chen-Huang, and Y-t Huang, arXiv:2012.15849.

[40] F. P. Bhat, D. Chowdhury, P. Haldar, A. Sinha, and A. Zahed (to be published).

[41] A. L. Guerrieri, A. Homrich, and P. Vieira, Dual S-matrix bootstrap. Part I. 2D theory, J. High Energy Phys. 11 (2020) 084. 